\title{
Smoking and periodontal diseases: patient awareness
}

Poor patient awareness of the relationship between smoking and periodontal diseases

Z. H. S. Lung, M. G. D. Kelleher, R.W.J. Porter, J. Gonzalez and R.F.H. Lung Br Dent J 2005; 199: 731-737

\section{Objective}

To investigate patients' knowledge of the effects of smoking on periodontal health.

Design

Patient answered questionnaires, which were anonymous.

Setting

Patients who attended GKT Dental Institute, King's College, London for dental treatment.

Subjects

One thousand patients attending Restorative Consultant Clinics and Primary Dental Care

Results

Seventy-eight per cent of patients were aware that smoking had a negative impact on health. However, $52 \%$ of these patients who were aware could not state what the negative effects were on oral health. Seven per cent of patients stated that smoking affected the gums but did not state how it affected the gums. Only 6\% of respondents knew specifically of the link between smoking and periodontal disease. The only statistically significant factors associated with increased awareness were smoking status, ie being a non-smoker and being registered with a general dental practitioner. Non-smokers and those registered with GDPs were more likely to be aware of the association between smoking and periodontal diseases.

Conclusions

This study highlights patients' lack of awareness of the relationship between smoking and periodontal diseases, with only $6 \%$ of respondents knowing of the link between tobacco and periodontal diseases.

\section{IN BRIEF}

- Provides evidence that smoking is a major risk factor in the progression of periodontitis.

- Only $6 \%$ of referred patients are aware of the damage smoking causes to their periodontal health.

- Emphasises the undervalued role of dental practitioners in providing smoking cessation counselling to their patients.

- Highlights the ineffectiveness of public health campaigns in educating patients that smoking increases the risks of their teeth 'falling out' by more than $300 \%$.

\section{COMMENT}

The role of tobacco in many diseases is well established and its major role in progression and severity of periodontal diseases has been highlighted.' This study shows that the public's understanding of this role is relatively poor, although most were aware of smoking having some impact on oral health.

The management of smoking related diseases costs the NHS $£ 1.5$ billion. The UK Government has launched vigorous campaigns to encourage the public to stop smoking or, especially in teenagers, to desist from taking up the habit - highlighting such factors as premature ageing and reduction in sex drive. Thus all health professionals are expected to advise patients of the impact of smoking and ensure that support for smoking cessation programmes tailored to individual patient's requirements is built into treatment strategies. The dental environment, with the patients being seen on a regular basis, offers opportunities to reinforce these messages in addition to emphasising the role of tobacco in oral health. Dentists should take opportunities to train in smoking cessation management, involve or train the whole team ${ }^{2}$ and build this into the supportive routine maintenance programmes for their patients.

Individual practices could conduct a survey similar to this one to establish the percentage of patients who attend regularly who smoke and consider setting targets to reduce this percentage. It would be interesting to know whether the local purchasers would offer financial incentives to practitioners for this purpose. The public health impact of even a small percentage giving up would be significant.

\section{R. Joshi, Consultant in Restorative Dentistry, Charles Clifford} Dental Hospital, Sheffield

1. Hujoel P P, del Aguila M A, DeRouen PA, Bergstrom J. A hidden epidemic during the 20th century? Community Dent Oral Epidemio/ 2003; 31: 1-6.

2. Johnson N W. The role of the dental team in tobacco cessation. Euro J Dent Educ 2004; 8 (Suppl 4): 18-24.

doi: $10.1038 /$ sj.bdj.4812972 